\title{
Duo complice : pharmaciens et techniciens en pharmacie
}

\author{
par Clarence Chant
}

$M$ algré les rôles reconnus des pharmaciens comme membres des équipes de soins de santé au sein des hôpitaux et dans la communauté, il y a une réelle pénurie de pharmaciens pour remplir ces rôles. Aux États-Unis, l'importance de cette pénurie a été modélisée à partir de diverses bases de données de services de pharmacie clinique et de personnel ${ }^{1}$. Le modèle a révélé que la mise en œuvre d'une série de cinq services de pharmacie clinique de base associés à des améliorations des résultats cliniques (c.-à-d., fournir de l'information sur les médicaments, prendre en charge les réactions indésirables aux médicaments, participer aux tournées, gérer les protocoles d'utilisation des médicaments et obtenir les histoires médicamenteuses) chez $100 \%$ des patients hospitalisés d'ici 2020 nécessiterait 14508 postes de pharmaciens équivalents temps plein supplémentaires! Bien que les effectifs au Canada n'ait pas fait l'objet d'une modélisation similaire, le récent rapport du projet Aller de l'avant : les futures ressources humaines en pharmacie, commandité par le gouvernement fédéral, semble faire écho à la pénurie qui sévit aux États-Unis ${ }^{2}$. Compte tenu de telles pénuries et de l'élargissement continu des rôles des pharmaciens comme fournisseurs de soins aux patients, le recours aux techniciens en pharmacie pour seconder les pharmaciens dans des tâches cliniques sélectionnées a été préconisé comme une solution potentielle, ne serait-ce que partielle.

Dans ce numéro du Journal, Johnston et ses collaborateurs ${ }^{3}$ font état d'une étude clinique aléatoire comparant les meilleurs schémas thérapeutiques possibles obtenus au service des urgences par les pharmaciens et les techniciens en pharmacie ${ }^{3}$. Dans leur étude, Johnston et ses collaborateurs ont découvert que les techniciens en pharmacie qualifiés étaient tout aussi efficaces que les pharmaciens pour obtenir les histoires médicamenteuses au service des urgences d'un hôpital communautaire et que le nombre de divergences constatées lors du bilan comparatif des médicaments subséquent pour des patients choisis au hasard était semblable. En outre, ces divergences étaient similaires en gravité, d'après l'évaluation d'un tiers. La puissance statistique de l'étude était insuffisante, comptant seulement 59 patients, et pour la majorité d'entre eux, on n'a constaté aucune divergence non intentionnelle de médicaments (47 et 50 patients selon les résultats respectifs des pharmaciens et des techniciens). Néanmoins, cette étude vient augmenter le nombre croissant de données qui appuient le rôle des techniciens en pharmacie dans cet aspect du processus lié au bilan comparatif des médicaments ${ }^{4,5}$.

Et pour pousser le sujet un peu plus loin, la chronique "Le pour et le contre » dans ce numéro présente un débat sur l'élargissement du rôle clinique des techniciens en pharmacie dans des secteurs où les pharmaciens sont absents ${ }^{6,7}$. Les deux côtés de l'argumentation semblent logiques et convaincants, et les auteurs soulèvent de nombreuses questions pertinentes. À l'appui du "pour " l'élargissement du rôle des techniciens ${ }^{6}$, on compte l'optimisation des effectifs, les compétences des techniciens et les données probantes actuelles (y compris l'étude de Johnston et coll. $\left.{ }^{3}\right)$. Les raisons appelant à la prudence du côté du "contre " ${ }^{7}$ sont le manque de collaboration, les perceptions négatives des parties prenantes, les préoccupations réglementaires et l'absence d'optimisation des rôles des techniciens, même dans les domaines de la distribution.

Le recours aux techniciens en pharmacie clinique dans les hôpitaux n'est certainement pas nouveau. Des rapports antérieurs ont décrit des tâches de pharmacie clinique effectuées par des techniciens, qui dépassaient largement la collecte d'histoires médicamenteuses, notamment les entrevues, la prestation de conseils et la consignation des interactions avec des patients dans une clinique d'anticoagulothérapie, tout cela sous la supervision d'un pharmacien ${ }^{8}$. Cette approche tranche fortement avec le rôle des techniciens dans certains hôpitaux, où ils ne distribuent aucun médicament sans la validation par un pharmacien ni ne saisissent d'ordonnances, ce qui est très loin des tâches usuelles de leurs homologues dans les pharmacies communautaires.

Je crois personnellement que la question qui se pose à la profession n'est pas de savoir si nous devrions travailler en collaboration avec les techniciens afin d'optimiser les soins pharmaceutiques pour nos patients, mais plutôt de savoir quelle est la meilleure façon de collaborer pour procurer les meilleurs avantages aux patients tout en minimisant les risques ${ }^{9}$. D’autres professions de la santé, comme les soins 
infirmiers et la médecine, ont déjà souscrit, à des niveaux différents, à ce concept de collaboration intraprofessionnelle avec du personnel de soutien pour améliorer les soins aux patients, et la profession de pharmacien ne devrait pas faire bande à part. En fait, le Bureau des examinateurs en pharmacie du Canada propose déjà un examen national pour les techniciens. En outre, du moins en Ontario, le collège des pharmaciens de cette province a élaboré un projet de normes de pratique pour les techniciens en pharmacie autorisés. Ces normes comprennent la collecte des histoires médicamenteuses, car cette tâche fait partie du programme de formation des techniciens ${ }^{10}$. Il faut admettre que les données à l'appui de la valeur ajoutée du rôle clinique du technicien en pharmacie sont provisoires (ce qui est également vrai pour certaines activités des pharmaciens), mais cela ne devrait pas nous empêcher de débattre ce sujet dans tous les services de pharmacie hospitalière.

Les espèces qui survivent ne sont pas les espèces les plus fortes, ni les plus intelligentes, mais celles qui sadaptent le mieux aux changements. [traduction du site Web www.evene.fr] - Charles Robert Darwin

[Traduction par l'éditeur]

\section{Références}

1. Bond CA, Raehl CL, Patry R. Evidence-based core clinical pharmacy services in United States hospitals in 2020: services and staffing. Pharmacotherapy 2004;24(4):427-440.

2. Comité de gestion. Aller de l'avant : Les futures ressources humaines en pharmacie. Rapport final. Ottawa $(\mathrm{ON})$ : Association des pharmaciens du Canada; septembre 2008. Publié au http://www.pharmacyhr.ca/ Articles/Fre/93.pdf, Consulté le 14 juillet 2010.

3. Johnston RM, Saulnier L, Gould O. Obtaining the best possible medication history in the emergency department: comparison between pharmacy technicians and pharmacists. Can J Hosp Pharm 2010;63(5): 359-366.
4. Leung M, Jung J, Lau W, Kiaii M, Jung B. Best possible medication history for hemodialysis patients obtained by a pharmacy technician. Can J Hosp Pharm 2009;62(5):386-391.

5. Remtulla S, Brown G, Frighetto L. Best possible medication history by a pharmacy technician at a tertiary care hospital. Can J Hosp Pharm 2009; 62(5):402-405.

6. Horon K, Hennessey T. Should pharmacy technicians provide clinical services or perform patient care activities in areas without a pharmacist? The "pro" side. Can J Hosp Pharm 2010;63(5):392-393.

7. Gorman S, Heukshorst S. Should pharmacy technicians provide clinical services or perform patient care activities in areas without a pharmacist? The "con" side. Can J Hosp Pharm 2010;63(5):393-395.

8. Weber E, Hepfinger C, Koontz R, Cohn-Oswald L. Pharmacy technicians supporting clinical functions. Am J Health Syst Pharm 2005;62(23):24662472.

9. Patel A, Ross B. Walgreens told to pay $\$ 25.8$ million over teen pharmacy tech's error; mother of three got pills ten times prescribed strength, resulting in massive stroke. ABC News: The Blotter; 2010 Mar 1. Publié au abcnews.go.com/Blotter/walgreens-told-pay-285-mil-teen-pharmacytechs/story?id=9977262. Consulté le 14 juillet 2010.

10. Ontario College of Pharmacists, Pharmacy Technician Working Group. Proposed standards of practice for registered pharmacy technicians. Toronto (ON): Ontario College of Pharmacists; Publié au www.ocpinfo.com/client/ocp/OCPHome.nsf/web/Proposed+ Technician+Standards. Consulté le 14 juillet 2010 .

Clarence Chant, Pharm. D., BCPS, FCSHP, FCCP, est spécialiste et leader en pharmacie clinique au St Michael's Hospital, à Toronto, en Ontario. $\|$ est également rédacteur adjoint du JCPH.

\section{Adresse de correspondance :}

Dr Clarence Chant

Pharmacy

St Michael's Hospital

30 Bond Street, Room Q4036

Toronto ON M5B 1W8

Courriel : chantc@smh.toronto.on.ca 